\title{
DURABILITY ASSESSMENT OF COMPOSITE STRUCTURAL ELEMENT REINFORCED WITH FABRIC DUE TO DELAMINATION
}

\author{
Jerzy Marszałek, Jacek Stadnicki \\ University of Bielsko-Biala, Department of Mechanical Engineering Fundamentals, Bielsko-Biala, Poland \\ ${ }^{*}$ Corresponding author. E-mail: jmarszalek@ath.bielsko.pl, jstadnicki@ath.bielsko.pl
}

\begin{abstract}
:
Layer composites reinforced with fabrics - laminates are construction materials in which mechanical properties can be shaped by designing their microstructure appropriately. However, the multi-phase microstructure of laminates makes it difficult to calculate the strength of the laminate constructions, especially when the reinforcement is a fabric. The article presents a special calculation model for determining the strength of an exemplary construction element made of laminate reinforced with a roving fabric with a plain weave made of carbon fibers. The computational model reflected in a simplified way the laminate microstructure, i.e. the number and orientation of the reinforcement fabric layers and its weave, and enabled a simulation of the behavior of the construction element under load up to fracture, which occurred as a result of breaking the reinforcement and interlayer crack-delamination. The simulation results were compared with the results of experimental stand tests. A method of modifying the computational model for laminates reinforced with non-plain weave was also suggested.
\end{abstract}

\section{Keywords:}

Layered composite, delamination, woven fabric, carbon fiber roving, finite element method

\section{Introduction}

Contemporary textile industry, apart from clothing and decorative textiles, also provides special textiles for technical use. Among them there are fabrics in which the warp and weft are rovings, i.e. bundles of parallelly arranged and untwisted glass, carbon or aramid fibers. Such fabrics, due to the high mechanical strength of the fibers, well-mastered and efficient weaving technology as well as consistency caused by the appropriate weave, are used as reinforcement in polymer layered structural composites - laminates. Reinforcement layers in laminates are impregnated with a matrix on the basis of polyester or epoxy resin and it is the type of fibers, fabric weave, number of layers and their mutual orientation and production technology (laminating) that determine whether the laminate will be an isotropic or anisotropic construction material [1].Laminates reinforced with fabrics made of fibers of very high strength are characterized by high specific strength, i.e. the strength related to density and that is why they are more commonly used in the production of parts of planes, cars, wind turbines and many other constructions [2,3,4]. Figure 1 shows the roving fabrics most often used in laminates. In the unidirectional fabric in Figure 1(a), the warp made of durable rovings is interwoven with a weft made of thin polyester roving of low strength, which is only to ensure fabric consistency and keep warp rovings parallel during lamination. A single layer of such reinforcement after impregnation with a matrix is durable practically in one direction - along the warp. In the fabric in Figure 1(b), the warp and weft are durable rovings connected by plain weave $\left(\frac{1}{1}\right.$, warp repeat $R_{\mathrm{p}}=2$, weft repeat $\left.R_{\mathrm{t}}=2\right)$, which ensures consistency during lamination. A single layer of such reinforcement after matrix impregnation is orthotropic, it has equal strength towards warp and weft. In the fabric in Figure 1 (c) the warp and weft of durable rovings are connected by a twill weave $\left(\frac{2}{2},(0,2,0), R_{\mathrm{p}}=4, R_{\mathrm{t}}=4\right)$. A single layer of such reinforcement is approximately orthotropic, but compared to plain weave fabric, the weft and warp are more loosely connected, which results in lower strength of the reinforcement layer than the previous one (Figure 1 (b)). It is worth adding that the fabric of reinforcement with a twill weave (Figure 1 (c)) during lamination is better laid on surfaces with a large curvature. The undoubted advantage of laminates reinforced with fabrics is the possibility to design their mechanical properties (strength and stiffness), which for the same fibers of reinforcement and matrix and the same lamination technology, depend on the weave used, the number of reinforcement layers and their mutual orientation. However, the result of the multiphase microstructure is that under the influence of excessive load in the laminate there might be cracking of the fibers of reinforcement and/or matrix, pulling out of the fibers from the matrix, breaking of the adhesive connection between the fiber and matrix, as well as breaking of the connection between layers of reinforcement - delamination, which is one of the most dangerous form of damage in laminated construction elements [5]. This is the reason why methods of modeling and simulating the work of construction elements under load which are known and verified for metals are not suitable for construction elements made of laminates. 
That is why the main aim of the article is to present a new proposal for modelling laminate construction elements using the formalism of the finite element method, which allows the determination of limit operating loads due to delamination and simulation of crack initiation and development, and at the same time:

- it takes into account the microstructure of the laminate, i.e. not only the number and orientation of layers but also the fabric weave of reinforcement,

- it makes it possible to determine crack initiation sites in the laminate and simulate their development,

- it uses the conditions and material constants which can be determined by means of a developed experimental and computational procedure based on easy-to-perform tests of specimens of a particular laminate, thanks to which it also takes into account the influence of laminate microstructure and laminating technology on its mechanical properties,

- it does not excessively increase the labour consumption of developing the computational model or the time of performing computer simulation.

Modeling and analyzing the strength of fabric reinforced laminates is most often carried out using the finite element method (FEM) because, compared to analytical methods, it allows one to take into consideration in one's analyses many factors determining the mechanical properties of laminates.

In commercial computational programs using FEM, fabricreinforced laminates are modeled at the macroscale level without reflecting their microstructure and, in particular, the weave of the reinforcement fabric (Figure 2 (a)). The model is built of finite elements of shell or solid type and the laminate microstructure is most often taken into consideration indirectly, replacing the laminate with a homogeneous material. This modeling method, due to good numerical efficiency of the model, can be used in the static analyses of structural elements made of laminates [6,7]. However, such a modeling method can cause erroneous simulation results $[7,8]$, and simulating delamination with its help requires the use of special computational methods (e.g. VCCT [9], cohesive zone method [10]), which significantly reduce the numerical efficiency of the model and require predicting the location of potential damage sites.

The strength of laminafte is determined by the strength of the fibers, matrix and fiber-matrix connection. In order to calculate it, FE models with different degrees of detail are used (Figure 2(b) and 2(c)). The reinforcement in the microscale model is modeled with finite elements of the solid type [11,12], which accurately reflect the cross-section and shape of individual fibers (Figure 2(b)). However, such models - due to a very large number of degrees of freedom, are not numerically effective. For this reason, microscale models are not used in engineering calculations of laminate construction elements. A reasonable compromise between macro and microscale models are mesoscale models (Figure 2(c)), which take into account - in a simplified way - the laminate microstructure resulting from the weave of the reinforcement fabric. That is why the mesoscale models are much more numerically effective compared to the microscale ones and, moreover, they do not require discretization of reinforcement rovings into single fibers (there is an assumption about roving homogenization [13]). Determining laminate strength then consists in checking the strength criteria for roving and matrix and for the connection between reinforcement and matrix $[14,15]$. Despite good numerical

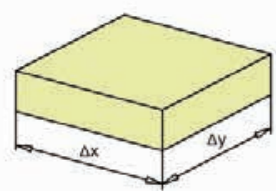

(a) Macroscale level

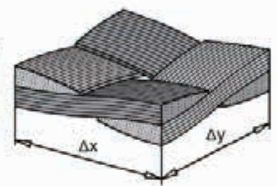

(b) Microscale level

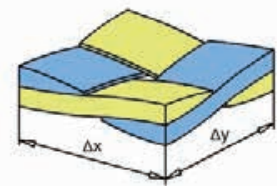

(c) Mesoscale level
Figure 2. Observation levels used in modeling plain weave reinforcement fabric

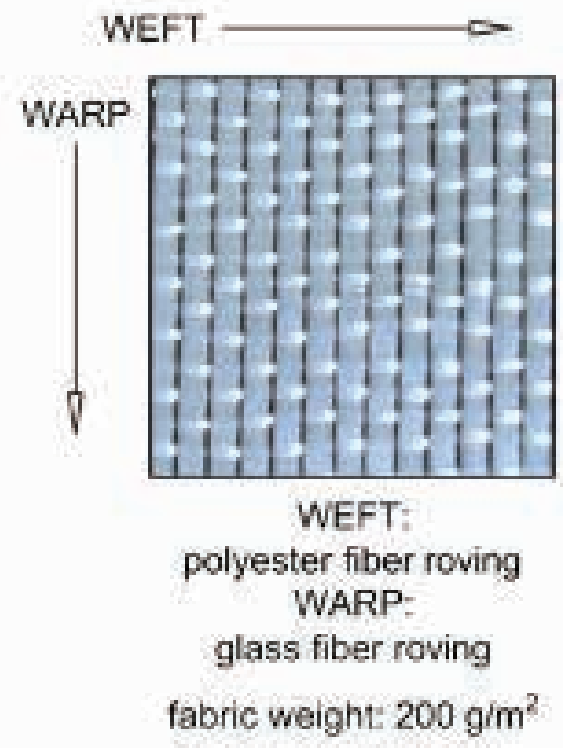

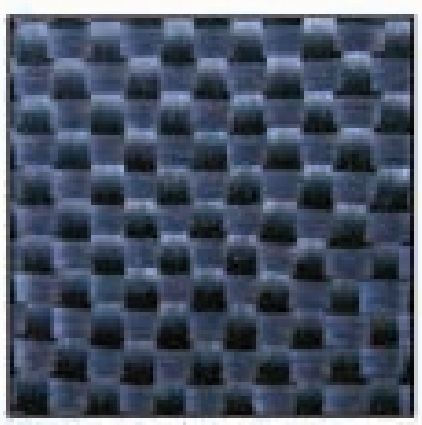

WEFT and WARP carbon fiber roving

fabric weight: $220 \mathrm{gim}^{2}$

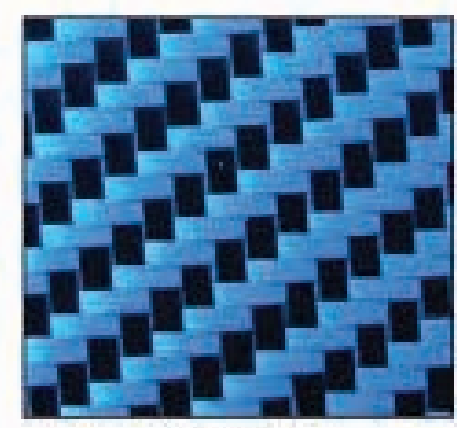

WEFT:

aramid fiber roving (blue) WARP: carbon fiber roving

fabric weight: $170{\mathrm{~g} / \mathrm{m}^{2}}^{2}$

Figure 1. Roving fabrics most often used as reinforcement in laminates 
efficiency, the mesoscale models described in the literature are not used in engineering practice for modeling delamination in laminate construction elements. Just like microscale models, they require the use of special computational methods for modeling cracking, which worsen their numerical efficiency and require the prediction of the location of potential crack sites.

\section{Computational FE model}

The method of modeling the matrix impregnated fabric (lamina) presented below assumes that the fiber rovings are reflected with finite elements of beam type and the matrix with elements of shell type. Figure 3 shows a model of the so-called unit cell, i.e. the smallest repetitive element of the microstructure (RUC - repetitive unit cell) extracted from a fabric with plain weave. RUC consists of two pairs of rovings interwoven with each other and a fragment of the matrix. In order to simplify the RUC model, it was assumed that dimension $\Delta z$ is small compared to dimensions $\Delta \mathrm{x}$ and $\Delta \mathrm{y}$. Then RUC is defined by four nodes lying in one plane at the roving interweaving sites $\mathrm{N}_{1}, \mathrm{~N}_{2}, \mathrm{~N}_{3}, \mathrm{~N}_{4}$. Beam elements $\mathrm{R}$ modeling matrix-impregnated roving sections (weft and warp) are characterized by two crosssectional parameters i.e. the area of cross section of roving $A_{\mathrm{R}}$ and the moment of inertia of cross-section $I_{R}$ because matriximpregnated roving, apart from stiffness of tension, also has stiffness of bending (it is not flabby). The matrix filling with the space between roving sections is reflected by a four-node shell element $\mathrm{S}$ with thickness $T_{\mathrm{S}}$.

The possibility to modify the mesoscale model of unit cell makes it useful in modeling laminates reinforced with fabric of a non-plain weave. For example, in the half basket weave, which is a derivative of plain weave, there are non-interwoven roving sections which are arranged parallelly on the surface of the fabric. An example of such a fabric $\left(\frac{2}{2},(0,1,0), R_{\mathrm{p}}=4, R_{\mathrm{t}}=2\right)$ and the corresponding mesoscale model are shown in Figure 4. The nodes at roving cross sites where the weft is under the warp are marked in white. Beam elements modeling the warp are stretched between all nodes towards axis 1. In turn, beam elements modeling weft are only stretched between the white nodes towards axis 2 . That is why in the single lamina model reinforced with half basket weave, longer beam elements modeling non-interwoven weft sections can be distinguished (weft beam elements are not attached to the nodes marked in black). This way of modeling the weft does not divide the weft into short sections between all nodes, which distinguishes this model from the plain weave fabric model (Figure 3 ). Then the neighboring nodes (white and black) can be connected with each other by means of four-node shell elements modeling the matrix. By rotating the coordinate system in Figure 4 by a right angle, one gets RUC for transverse half basket weave $\left(\frac{2}{2}\right.$, $\left.(0,1,0), R_{\mathrm{p}}=2, R_{\mathrm{t}}=4\right)$ and RUC superposition for longitudinal and transverse half basket weave will create RUC for twill weave $\left(\frac{2}{2},(0,2,0), R_{\mathrm{p}}=4, R_{\mathrm{t}}=4\right)$.

\section{Strength assessment with reference to delamination of an exemplary construction element}

The usefulness of the mesoscale model (Figure 3) for assessing the strength of construction elements due to delamination was tested on the example of a laminate reinforcement beam for side doors of a passenger car (Figure 5). It is a construction element which protects passengers and absorbs part of the side impact energy by being deformed elastically and then destroyed. The beam was made of eight layers of roving fabric (3k CF200 style450), stacked on top of each other, with a plain weave of carbon fibers whose warp was parallel to the axis of the beam. The reinforcement layers during lamination were impregnated with a chemically hardenable polymer matrix based on epoxy resin Biresin ${ }^{\circ}$ CR120. The door beam is an example of a construction element of an irregular and complex shape, because its outline is an irregular curve and the shape is made of flat and curved surfaces. The strength of the door beams is checked in a standard way using the three-point bending test (Figure 6). The construction of the measuring stand and the test procedure are described in detail e.g. in

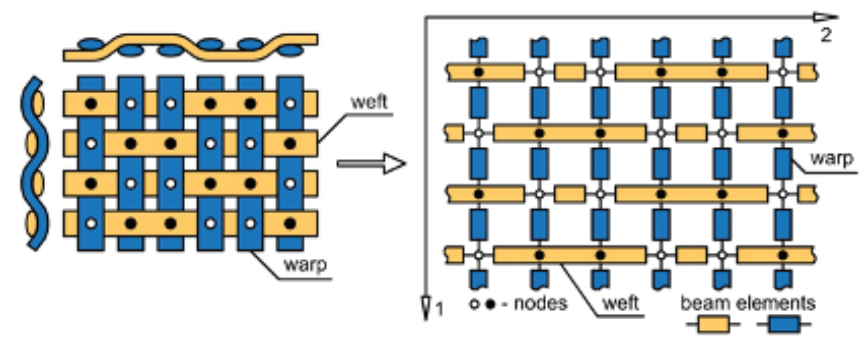

Figure 4. Arrangement of beam elements for the fabric with half basket weave

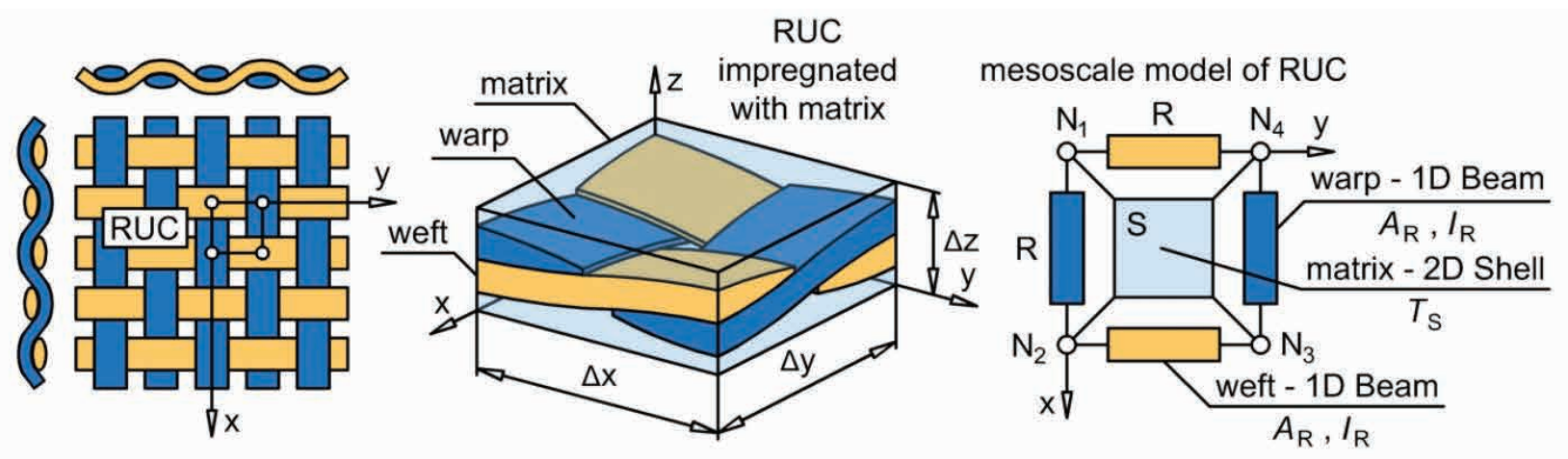

Figure 3. Mesoscale model of RUC for lamina reinforced with roving fabric of plain weave 
GMW16418 standard [16]. As a result of the test, $F_{s}\left(f_{s}\right)$ curve is obtained, i.e. the course of the push force of bending pin on beam $F_{\mathrm{s}}$ as a function of beam deflection under bending pin $f_{\mathrm{s}}$, on the basis of which the door beam strength is assessed.

The construction of the beam mesoscale model began with the preparation of the geometric shell model, which was then discretized by means of four-node shell finite elements. The discretization concerned the external surface of the modeled beam. Then the nodes of the shell model were parallelly shifted by the thickness of the layer, thus building up the nodes of all other reinforcement layers. In the meshes of nets created in this way in the layers, beam elements $R$ and shell elements $S$ were generated, forming RUC in accordance with the diagram in Figure 7. In the next stage, beam elements $P$ were inserted between appropriate nodes of adjacent layers, with matrix material constants and substitute cross-sectional parameters $A_{\mathrm{P}}$ and $I_{\mathrm{P}}$ modeling connections between the layers (Figure 7).

The matrix material constants necessary for building the model were read from the technical card [17]. Due to the lack of data on the fabric, a roving tensile test was carried out, on the basis of which a substitute Young's modulus of roving $\left(E_{R} \cong 105 \mathrm{GPa}\right)$

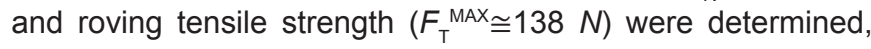
assuming that the cross-section of the roving is uniform. Then, the values of cross-sectional parameters $\left(A_{\mathrm{P}}=A_{\mathrm{P}(0)}\right.$ and $\left.T_{\mathrm{S}}=T_{\mathrm{S}(0)}\right)$ were determined for finite elements modeling RUC (Table 1) keeping their volume share of the finite element in RUC. In order to determine the cross-sectional parameters $I_{R}=I_{R(0)}$ and $I_{P}=I_{P(0)}$, the calibration of the laminate mesoscale model was performed with the use of parametric optimization. The objective function was the best compatibility of two bending lines of a rectangular specimen made of the same laminate and

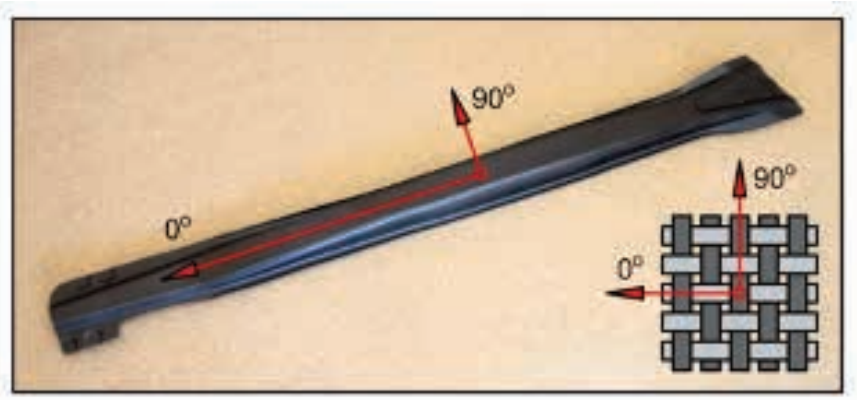

Figure 5. Laminate door beam with marked orientation of reinforcement fabric

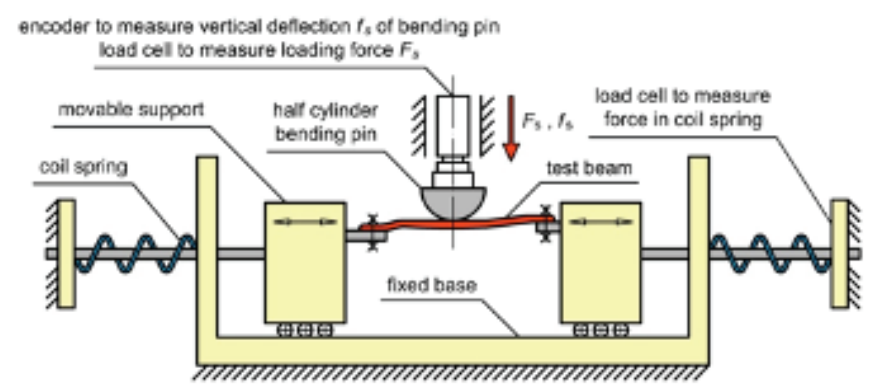

Figure 6. Schematic of the door beam testing stand according to the standard [16] in the same technology as the modeled beam, in the three-point bending test. One bending line was determined experimentally and the other as a result of simulation of specimen FE model. The model calibration procedure was described in detail on the example of another mesoscale model in work [7].

The cross-sectional area $A_{\mathrm{R}}$ of roving made of carbon fibers was determined on the basis of weight analysis. Twenty sections of rovings were taken from the fabric. Each $1000 \mathrm{~mm}$ long section was weighed with the accuracy to $0.001 \mathrm{~g}$ and then the result was averaged. Knowing the length and average mass of each section and the density of carbon fibers, the average cross-sectional area of the roving was determined $\left(A_{\mathrm{R}}=A_{\mathrm{R}(0)}=0.12 \pm 0.01 \mathrm{~mm}^{2}\right)$.

In order to assess the resistance of the beam to delamination, criteria for the strength of the finite elements forming RUC (Figure 3 ) had to be defined. It was assumed that during simulation of delamination (initiation and propagation of crack between layers) the stiffness (cross-sectional parameters) of finite elements creating connections between layers $\left(A_{\mathrm{P}(0)}, I_{\mathrm{P}(0)}\right)$ will be reduced and/or these elements will be removed if the appropriate strength criterion is exceeded.

The criteria were defined resulting from three possible basic modes of laminate fracture as a result of initiation of crack between layers, i.e. tension mode, sliding shear mode and scissoring shear mode [18]. Beam element $P$, in which the threshold value of tensile or shear force was exceeded, was searched and then, depending on the existing fracture mode, removed in one step (tension mode) or its stiffness was reduced similarly to beam elements $\mathrm{R}$ (sliding and scissoring shear mode). The method of determining the threshold values of tensile and shear forces for beam elements $P$ for three fracture modes is presented in work [18]. The task was to carry out simulation of experimental tests of delamination of pre-delaminated specimens of laminate in the conditions of the following tests: DCB (double cantilever beam), ENF (end notched flexure) and MECT (modified edge crack torsion). Comparing the experimental curves and the ones determined during the simulation of experiments, the threshold values of forces in finite elements $\mathrm{P}$ modeling connections between layers corresponding to the moment of crack initiation were read. Yet, for the DCB test it was the tensile force $F_{\mathrm{P}(0)}{ }^{z}$ in the finite element and for the ENF and MECT tests these were shear forces $F_{\mathrm{P}(0)}{ }^{\mathrm{x}}$ in the finite elements (Figure 7). In the case

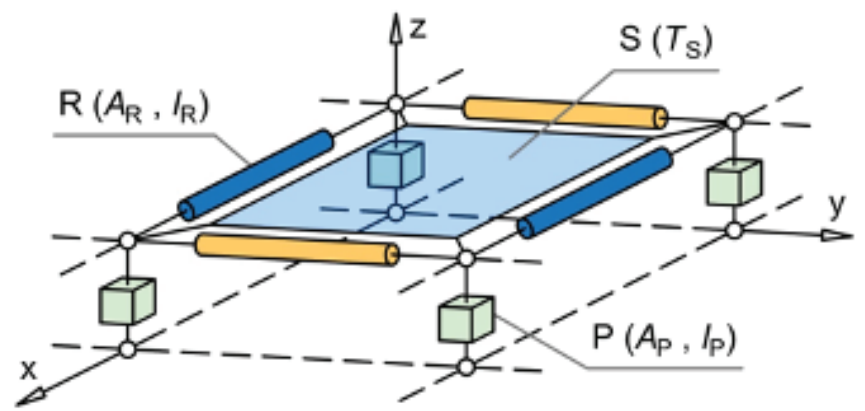

Figure 7. RUC diagram with elements modeling connections between adjacent layers of reinforcement 
Table 1. Values of parameters of finite elements creating RUC model

\begin{tabular}{|c|c|c|c|}
\hline Finite element type & Area of cross section & Area moment of inertia & Thickness \\
\hline 1D Beam - P & $A_{\mathrm{P}(0)}=1.56 \mathrm{~mm}^{2}$ & $I_{\mathrm{P}(0)}=0.2 \mathrm{~mm}^{4}$ & - \\
\hline 1D Beam - R & $A_{\mathrm{R}(0)}=0.12 \mathrm{~mm}^{2}$ & $I_{\mathrm{R}(0)}=0.065 \mathrm{~mm}^{4}$ & - \\
\hline 2D Shell $-\mathrm{S}$ & - & - & $T_{\mathrm{S}(0)}=0.04 \mathrm{~mm}$ \\
\hline
\end{tabular}

of the DCB test, the procedure of removing beam elements $\mathrm{P}$ after exceeding the threshold value $F_{\mathrm{P}(0)}{ }^{\mathrm{x}}$ of the tensile force in one step enabled good reflection of the results of the experimental tests. However, this course of action did not work for ENF and MECT tests. To improve the compliance of results another procedure was conducted, consisting in gradual reduction of the cross-sectional area $A_{P(0)}$ of beam elements $\mathrm{P}$ in several steps according to the exponential relation. Such a procedure of $A_{\mathrm{P}(0)}$ cross-section reduction in seven steps ensured very good consistency of the results of the simulation and experiment (the beam element was removed in the eighth step) [18]. It was assumption that cross-section $A_{P(i)}$ in the succeeding steps of reduction in relation to the initial section is determined by a term of geometrical progression:

$$
A_{\mathrm{P}(i)}=0.55^{i} \cdot A_{\mathrm{P}(0)} \quad \text { for } \quad i=1,2, \ldots, 7
$$

Such a procedure also required determining the threshold values of the shear force $F_{\mathrm{P}(\mathrm{i})}$ in the succeeding steps of section reduction, which is described by the geometrical progression:

$$
F_{\mathrm{P}(i)}=0.78^{i} \cdot F_{\mathrm{P}(0)}^{\mathrm{x}} \text { for } i=1,2, \ldots, 7
$$

In addition, an assumption was made that the destruction of the bending door beam can also take place as a result of a fracture of rovings forming the reinforcement fabric. That is why a special procedure was developed to simulate the crack of matrix-impregnated roving, which was implemented in the model by gradual reduction of stiffness (of cross-sectional parameters $A_{\mathrm{R}}, I_{\mathrm{R}}$ ) of the beam elements $\mathrm{R}$ modeling roving sections. For this purpose, the results of experimental tests were used, in which three-point bending of rectangular composite specimen was performed and individual roving sections were tensioned. On the basis of the bending test of the composite specimen, the curve of force $F_{B}$ was determined as a function of the deflection of specimen $f_{\mathrm{B}}$ and the value of the deflection $f_{\mathrm{B}}^{\mathrm{NL}}$ was identified, after exceeding of which the curve changed from the linear part into a non-linear one. By performing the simulation of the specimen model bending for the deflection corresponding to the value of $f_{\mathrm{B}}^{\mathrm{NL}}$, the value of tensile force $F_{R} \cong 125 N$ in the beam elements $R$ at the place of potential damage was read. In the case of the tensile test of individual sections of roving, tensile force $F_{\mathrm{T}}{ }^{\mathrm{NL}}=F_{\mathrm{R}}{ }_{\mathrm{R}} \cong 134 \mathrm{~N}$ was determined, at which the process of roving destruction began, as well as force $F_{\mathrm{T}}{ }^{\mathrm{MAX}}=F_{\mathrm{R}}{ }_{\mathrm{R}}{ }^{\mathrm{II}} \cong 138 \mathrm{~N}$, after exceeding of which the rovings were broken.
On the basis of many numerical experiments, it was established that the procedure of reducing the stiffness of finite elements $\mathrm{R}$ modeling the roving sections in which force $F_{\mathrm{R}}$ exceeded the threshold value $F_{R}{ }^{(1)}=F_{R}{ }^{l}=125 N$ took place in two phases. In the first phase, the bending stiffness of the finite element was reduced in three steps, which went down to reducing the moment of inertia of cross-section $I_{R(0)}$ to a value close to zero (the roving section $\mathrm{R}$ became flabby) in accordance with formulas (4), (5) and (6). However, each reduction of the moment of inertia of the cross-section was accompanied by an increase in the initial threshold value of force $F_{\mathrm{R}}{ }^{(0)}$ in accordance with formula (7).

$$
\begin{gathered}
\text { if } F_{\mathrm{R}} \leq F_{\mathrm{R}}{ }^{\mathrm{I}(0)} \text { then } I_{\mathrm{R}}=I_{\mathrm{R}(0)} \\
\text { if } F_{\mathrm{R}}{ }^{\mathrm{I}(0)} \leq F_{\mathrm{R}} \leq F_{\mathrm{R}}{ }^{\mathrm{I}(1)} \text { then } I_{\mathrm{R}}=I_{\mathrm{R}(1)}=0.25 \cdot I_{\mathrm{R}(0)} \\
\text { if } F_{\mathrm{R}}{ }^{\mathrm{I}(1)} \leq F_{\mathrm{R}} \leq F_{\mathrm{R}}{ }^{\mathrm{I}(2)} \text { then } I_{\mathrm{R}}=I_{\mathrm{R}(2)}=0.25 \cdot I_{\mathrm{R}(1)} \\
\text { if } F_{\mathrm{R}} \geq F_{\mathrm{R}}{ }^{\mathrm{I}(2)} \text { then } I_{\mathrm{R}}=I_{\mathrm{R}(3)} \cong 0 \\
F_{\mathrm{R}}{ }^{\mathrm{I}(1)}=\alpha \cdot i+F_{\mathrm{R}}{ }^{\mathrm{I}(0)} \text { where } \alpha=\frac{F_{\mathrm{R}}^{\mathrm{II}}-F_{\mathrm{R}}^{\mathrm{I}}}{3}, i=1,2
\end{gathered}
$$

In the second phase, after exceeding the threshold value of force $F_{\mathrm{R}}{ }^{\mathrm{A}(0)}=F_{\mathrm{R}}{ }^{\mathrm{I}}=134 \mathrm{~N}$, the tensile stiffness of finite element was reduced in three steps, which went down to reducing cross-section $A_{\mathrm{R}(0)}$ to a value close to zero (the roving section was broken) in accordance with formulas (10), (11) and (12). However, each cross-section reduction was accompanied by an increase in the initial threshold value of force $F_{\mathrm{R}}^{\mathrm{A}(0)}$ in accordance with formula (8).

$$
\begin{aligned}
& F_{\mathrm{R}}{ }^{\mathrm{A}(\mathrm{i})}=\beta \cdot i+F_{\mathrm{R}}{ }^{\mathrm{A}(0)} \text { where } \beta=\frac{F_{\mathrm{R}}^{I I I}-F_{\mathrm{R}}^{\mathrm{II}}}{2}, i=1,2 \\
& \text { if } F_{\mathrm{R}} \leq F_{\mathrm{R}}{ }^{\mathrm{A}(0)} \text { then } A_{\mathrm{R}}=A_{\mathrm{R}(0)} \\
& \text { if }{F_{\mathrm{R}}}^{\mathrm{A}(0)} \leq F_{\mathrm{R}} \leq F_{\mathrm{R}}{ }^{\mathrm{A}(1)} \text { then } A_{\mathrm{R}}=A_{\mathrm{R}(1)}=0.90 \cdot A_{\mathrm{R}(0)} \\
& \text { if }{F_{\mathrm{R}}}^{\mathrm{A}(1)} \leq F_{\mathrm{R}} \leq F_{\mathrm{R}}{ }^{\mathrm{A}(2)} \text { then } A_{\mathrm{R}}=A_{\mathrm{R}(2)}=0.90 \cdot A_{\mathrm{R}(1)} \\
& \text { if } F_{\mathrm{R}} \geq F_{\mathrm{R}}{ }^{\mathrm{A}(2)} \text { then } A_{\mathrm{R}}=A_{\mathrm{R}(3)} \cong 0
\end{aligned}
$$

Constants in formulas (1) and (2) as well as ways of reducing the moment of inertia of section $I_{\mathrm{R}(0)}$ and section $A_{\mathrm{R}(0)}$ during delamination expressed by formulas (3), (4),..., (12) were determined according to the described procedure on the basis of experimental tests of specimens of laminate made using a 
specific Biresin $®$ CR120 resin by wet lamination technology in a closed double-sided mould. If the laminate was manufactured using a different resin or a different lamination technology (e.g. wet lamination in a one-sided mould or lamination using prepregs and heating in an autoclave), such a procedure would have to be repeated to obtain constant values and the course of reducing the moment of inertia of section $I_{\mathrm{R}(0)}$ and section $A_{\mathrm{R}(0)}$, which would ensure the compatibility of simulation and experiment results. It is worth mentioning that the experimental tests of delamination of laminate specimens, which are part of the mentioned procedure, are easy to perform and require basic laboratory equipment.

Simulation of the three-point bending test of the laminate door beam, in which the proposed mesoscale FE model and the procedures for initiating and propagating interlayer cracks were used in practice in ANSYS program using internal APDL command language. APDL language was also used to develop a procedure for automated generation of a mesoscale door beam model.

\section{Experimental verification}

On the basis of the simulation results exported by Ansys program, the force-deflection curve $F_{s}\left(f_{s}\right)$ was determined for the model of the analyzed beam, which was shown in Figure 8. The curve consists of three approximately linear fragments with different slopes, between which a slight decrease in force $F_{\mathrm{s}}$ occurs. The occurring decreases in force $F_{\mathrm{s}}$ were the result of the formation of local cracks in the model, the locations of which are shown in Figure 9. The first crack, marked with letter $A$, appeared on the edge of the support. The result of this crack was the first decrease in the force $F_{\mathrm{s}}$ of bending pin on the computational curve-point $\mathrm{NL}_{1(\mathrm{~m})}$ (Figure 8). The second decrease in the force at $\mathrm{NL}_{2(\mathrm{~m})}$ point was caused by crack $\mathrm{B}$. The largest crack $C$ was created at a distance of about $100 \mathrm{~mm}$ from cracks $A$ and $B$, which was recorded on the computational curve in the form of a clear decrease in the force $F_{s}$ at point $\operatorname{MAX}_{(m)}$. In the case of each of the cracks, disintegration of the microstructure of the model was similar. First, due to exceeding the threshold value of the tensile force, the stiffness of beam elements $\mathrm{R}$ modeling roving was reduced. Of the three possible delamination modes, sliding shear mode was dominant. With increasing disintegration of the unit cells, the tensile stress in the shell elements $\mathrm{S}$ modeling the matrix increased.

The assessment of the beam resistance to delamination was then verified in the experimental three-point bending test on a special stand (Figure 6), which met the requirements of the standard [16]. The door beam was screwed to the sliding supports which were connected to the fixed base of the stand by means of coil springs reflecting the stiffness of the car door frame. During the test, vertical displacement $f_{\mathrm{s}}$ of bending pin was applied at a speed of $2 \mathrm{~mm} / \mathrm{s}$ and the force $F_{\mathrm{s}}$ of bending pin on the beam was measured.

As a result of the conducted test, the curve of the force $F_{\mathrm{s}}$ as a function of the beam deflection $f_{\mathrm{s}}$ under the bending pin was obtained, which was compared with the curve determined during model simulation (Figure 8). On the experimental curve it is also possible to distinguish three approximately linear fragments with different slopes, between which there was a slight decrease in the value of force $F_{s}$. The decreases in force $F_{\mathrm{s}}$ occurred as a result of the formation of three cracks which appeared in the same places and in the same order as during the simulation (Figure 10). That is why three points were marked on the experimental curve (Figure 8): point $\mathrm{NL}_{1}$, where crack $A$ propagation began, point $\mathrm{NL}_{2}$, where propagation of crack $B$ began, and point MAX, after exceeding of which crack C was formed.

Table 2 summarizes the errors of force and deflection resulting from the comparison of calculated values with experimentally measured values. On their basis, relative errors of force and deflection were determined, relating absolute error values to measured values. The largest error of force was $8 \%$ and of deflection $4 \%$, which - given the complexity of the modeled delamination phenomenon - indicates very good compliance of the simulation and experiment results. As an additional measure of the quality of the fit of the model to the results of experimental tests, one can propose $R^{2}$ coefficient of determination, which was determined for the initial (linear) fragments of the curves (Figure 8), i.e. for the range $0-\mathrm{NL}_{1(\mathrm{~m})}$. The coefficient of determination was 0.92 , which further confirmed that the model reflects the real construction element of laminate very well.

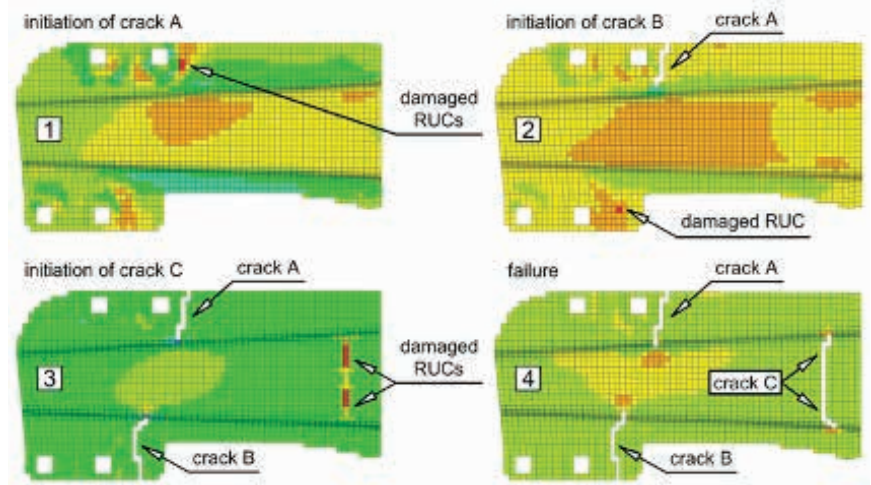

Figure 9. The order of formation and location of cracks in the beam mesoscale model
Figure 8. Computational and experimental force-deflection curves of side door beam

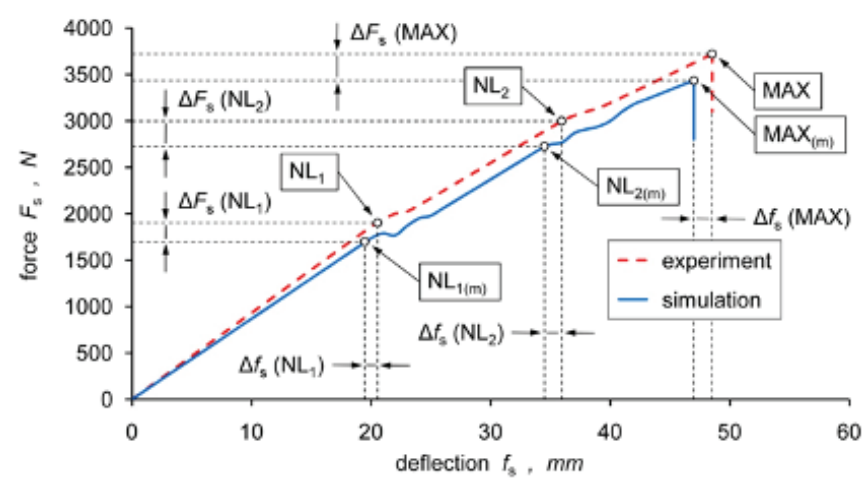

http://www.autexrj.com/ 
Table 2. Errors of deflection and bending force for mesoscale model

\begin{tabular}{|c|c|c|c|c|}
\hline Points & $\begin{array}{c}\text { Error of deflection } \\
\boldsymbol{\Delta \boldsymbol { f } _ { s }}, \boldsymbol{m m}\end{array}$ & $\begin{array}{c}\text { Relative } \\
\text { error of deflection } \\
\boldsymbol{\varepsilon} \boldsymbol{f}_{s}, \boldsymbol{\%}\end{array}$ & $\begin{array}{c}\text { Error of force } \\
\boldsymbol{\Delta} \boldsymbol{F}_{s}, \boldsymbol{N}\end{array}$ & $\begin{array}{c}\text { Relative } \\
\text { error of force } \\
\boldsymbol{\varepsilon} \boldsymbol{F}_{s}, \boldsymbol{\%}\end{array}$ \\
\hline $\mathrm{NL}_{1}$ and $\mathrm{NL}_{1(\mathrm{~m})}$ & 0.87 & 4.24 & 156.12 & 8.42 \\
\hline $\mathrm{NL}_{2}$ and $\mathrm{NL}_{2(\mathrm{~m})}$ & 0.93 & 2.59 & 234.59 & 7.87 \\
\hline${\mathrm{MAX} \text { and } \mathrm{MAX}_{(\mathrm{m})}}$ & 1.51 & 3.11 & 284.21 & 7.64 \\
\hline
\end{tabular}

Another fact confirming the usefulness of the proposed mesoscale FE model of a construction element made of fabricreinforced laminate is the comparison of the sites of formation of subsequent cracks during the bending test. As can be seen in Figure 10, during the simulation and the experiment the cracks formed at the same sites.

\section{Conclusions}

The proposed method of assessing strength with reference to delamination and stiffness of construction elements made of laminates reinforced with technical fabric, which uses the RUC concept proposed in the article and the mesoscale level of laminate microstructure observation, enables:

- simplified reflecting of the laminate microstructure, in particular of the weave of the reinforcement fabric and the number and orientation of layers;

Experimental tests of laminate specimens and construction elements indicate that their mechanical properties, including delamination resistance, depend on the weave of the reinforcement fabric. The comparison of the results of delamination simulation with the results of experimental tests of an exemplary laminate construction element - a door beam - confirmed that the method of modelling the laminate microstructure described in the article combined with experimental tests of specimens ensures good consistency of the results in terms of deflections and of crack initiation sites as well as limit values of loads that cause them.

- experimental determination of delamination strength criteria for a laminate with a specific microstructure from specific components produced by a specific lamination technology;

Although the necessity to carry out this procedure limits the use of the numerical values in the proposed criteria of laminate
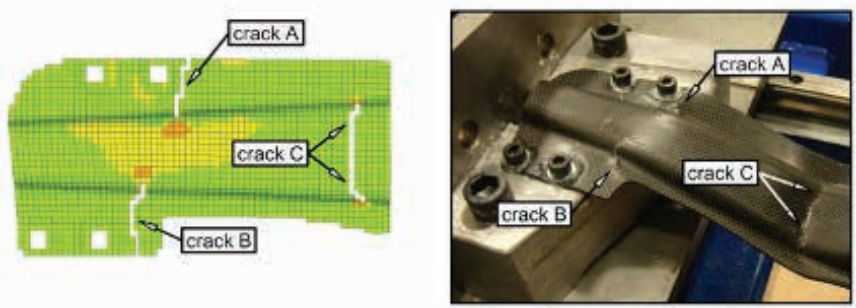

Figure 10. Indication of crack formation sites in the beam model after finishing bending simulation and in the beam destroyed experimentally resistance to delamination to specific cases, it ensures good consistency of simulation and experiment results. The proposed experimental and computational procedure for determining these criteria can be easily carried out for other laminates and other laminating technology with only basic laboratory equipment.

- simulation of delamination without the need to predict the location of crack formation and propagation;

Methods which are often used during the cumulation of cracking, such as VCCT or cohesive zone method, require predicting the potential crack initiation sites, which in engineering calculations can be difficult and sometimes even impossible. Removing this obstacle significantly broadens the scope of application of the method of modelling laminate construction elements described in the article.

- assessment of strength to delamination of undamaged laminate construction elements as well as determination of permissible loads for partially damaged elements (with small cracks), which can be further exploited under lower load.

The experimental verification of the mesoscale FE model of a real construction element made of laminate with an irregular and complex shape carried out on the example of a threepoint bending test of a passenger car door beam showed full usefulness of the proposed model in engineering calculations. It is worth adding that the authors' experience shows that thanks to the automation of generating mesoscale FE model, e.g. while using the APDL language of the ANSYS program, the time of developing a laminate construction element model is comparable to the time of developing a model of this element made of sheet metal.

\section{References}

[1] Carlsson, L. A., Adams, D. F., Pipes, R. B. (2014). Experimental characterization of advanced composite materials (4 ed.). CRC Press (Boca Raton).

[2] Xiao, Z., Pei, L., Zhang, F., et al. (2019). Parameter measurement of biaxial braided composite preform based on phase congruency. Autex Research Journal, 19(1), 8-16.

[3] Yang, Y., Liu, X., Wang, Y-Q., et al. (2017). A progressive damage model for predicting damage evolution of laminated composites subjected to three-point bending. Composites Science and Technology, 151, 85-93. 
[4] Dong, K., Peng, X., Zhang, J., et al. (2017). Temperaturedependent thermal expansion behaviors of carbon fiber/epoxy plain woven composites: Experimental and numerical studies. Composite Structures, 176, 329-341.

[5] Lindgaard, E., Bak, B. L. V. (2019). Experimental characterization of delamination in off-axis GFRP laminates during mode I loading. Composite Structures, 220, 953-960.

[6] Nour, A., Gherbi, M. T., Tawfiq, I. (2017). Analysis of the Bauschinger effect on a multilayer helicopter blade by XFEM simulation. Aerospace Science and Technology, 69, 97-113.

[7] Stadnicki, J., Tokarz, Z. (2016). Mesoscale finite element model for calculating deformations of laminate composite constructions. Advances in Mechanical Engineering, 8(2), 1-9.

[8] Vinšová, L., Urban, T. (2017). Testing of mechanical properties of thick-walled carbon fiber composite for FEM simulations. Materials Today: Proceedings, 4, 5989-5994.

[9] Krueger, R. (2004). Virtual crack closure technique: history, approach, and applications. Applied Mechanics Reviews, 57(2), 109-143.

[10] Lindgaard, E., Bak, B. L. V., Glud, J. A., et al. (2017). A user programmed cohesive zone finite element for ANSYS Mechanical. Engineering Fracture Mechanics, 180, 229239.
[11] Soni, G., Singh, R., Mitra, M., Falzon, B. G. (2014). Modelling matrix damage and fibre-matrix interfacial decohesion in composite laminates via a multi-fibre multi-layer representative volume element (M2RVE). International Journal of Solids and Structures, 51, 449-461.

[12] Naghdinasab, M., Farrokhabadi, A., Madadi H. (2018). A numerical method to evaluate the material properties degradation in composite RVEs due to fiber-matrix debonding and induced matrix cracking. Finite Elements in Analysis and Design, 146, 84-95.

[13] Bednarcyk, B.A., Stier, B., Simon, J-W., et al. (2015). Meso- and micro-scale modeling of damage in plain weave composites. Composite Structures, 121, 258-270.

[14] Doitrand, A., Fagiano, C., Irisarri, F.-X., Hirsekorn, M. (2015). Comparison between voxel and consistent mesoscale models of woven composites. Composites: Part $A$, 73, 143-154.

[15] Esposito, L., Pucillo, G. P., Penta, F., Rosiello, V. (2016). Micromechanical study and simulation of the interlaminar failure of a woven composite laminate. Procedia Structural Integrity, 2, 1870-1877.

[16] GMW16418: 2016. Door beam bending strength test.

[17] Product data sheet: 2014. Biresin ${ }^{\circledR}$ CR120. Composite resin system.

[18] Marszałek, J., Stadnicki, J. (2018). Simulation of delamination in a composite using a shell-beam mesoscale finite element model. Fibres \& Textiles in Eastern Europe, 4(130), 97-103. 